\title{
The Processing of Two-Digit Numbers Depends on Task Instructions
}

\author{
Bert Reynvoet, ${ }^{1}$ Karolien Notebaert, ${ }^{1}$ and Eva Van den Bussche ${ }^{2}$ \\ ${ }^{1}$ Department of Psychology, University of Leuven, Belgium, ${ }^{2}$ Department of Psychology, \\ Brussels University, Belgium
}

\begin{abstract}
Recently, a lot of research has focused on resolving whether two-digit numbers are processed holistically or compositionally. This has led to inconsistent results. In the present study we investigated effects of task instructions. Subjects performed magnitude or parity judgments on targets preceded by masked primes containing parts of the target at a task-congruent (3\#_37) or task-incongruent (\#3_37) position. Priming effects were influenced by the instructions: In the magnitude task, the priming effects were primarily mediated by the congruency of the decade digit, whereas in the parity task they were elicited by the congruency of the unit digit, which is in line with a flexible compositional processing style. These and previous findings show that two-digit numbers can be processed in a very flexible way, depending on the task context.
\end{abstract}

Keywords: two-digit numbers, priming, position-specific effects, task-dependent processing

Recently, researchers have discussed whether two-digit numbers are represented as holistic values or whether they are decomposed into a decade number and a unit number. Evidence for a holistic representation stems from comparison tasks (e.g., Dehaene, Dupoux, \& Mehler, 1990) and priming tasks (e.g., Reynvoet \& Brysbaert, 1999). For instance, Reynvoet and Brysbaert showed that the priming distance effect (i.e., slower reaction times (RTs) for increasing numerical distances between prime and target) is equal for prime-target pairs within the same decade (e.g., prime " 8 " target "9") as for pairs from a different decade (e.g., prime " 10 " target "9") for a constant numerical distance. In contrast, other studies have shown that two-digit numbers can be decomposed as well (e.g., Gazzellini \& Laudanna, 2011 (this issue); Nuerk, Weger, \& Willmes, 2001; Verguts \& De Moor, 2005). For instance, Nuerk et al. (2001) showed that comparisons are faster for pairs where the larger number also contains the largest unit number (e.g., 23_37) than for pairs where this is not the case (e.g., 29_43), an effect known as the unit-decade compatibility effect. Recently, a number of studies have provided evidence for both types of representation, holistic and compositional, depending on experimental factors. For instance, a comparison experiment of Zhang and Wang (2005) showed that the representation of two-digit numbers depends on whether the referent number is presented simultaneously (external representation) or has to be kept in memory (internal representation). An external representation resulted in a compositional processing style, whereas an internal representation in a holistical processing style. More recently, Zhou, Chen, Chen, and Dong (2008) observed that, when double digits in a number-matching task were presented simultaneously, participants applied a compositional processing style. In contrast, when the numbers were presented serially, they were processed holistically. In line with these results, a study of Ganor-Stern, Pinhas, and Tzelgov (2009) provided further support for a compositional representation when two numbers in a comparison task were presented simultaneously, whereas both numbers were found to be processed holistically when they were presented sequentially. However, the latter was only the case when both decade and unit were relevant for the task at hand (Experiment 2). In contrast, Moeller, Nuerk, and Willmes (2009) found that compositional processing of two-digit numbers does not rely on external representations, but can also be observed whenever an internal representation of the numbers is involved.

The aim of the present study is to investigate whether the processing of two-digit numbers also depends on the informative value of both components of a two-digit number. Whereas previous studies have used the spatial numerical association of response codes (SNARC) effect (Ganor-Stern et al., 2009; Moeller et al., 2009) to investigate the processing of two-digit numbers, the present study used masked priming. Recently, it has been argued that the priming paradigm is a purer method to study number representations because it is not confounded by response processes (Van Opstal, Gevers, De Moor, \& Verguts, 2008). Moreover, short stimulus onset asynchronies between prime and target and masks ensure that the priming effects are automatic (Cohen-Kadosh \& Walsh, 2009; Reynvoet \& Notebaert, 2009). More specifically, in our experiment, two-digit target numbers were preceded by primes containing parts of the target at a task-congruent (3\#_37) or a task-incongruent (\#3 37) position. The primes were presented very briefly 
and were visually masked to eliminate strategic use of the primes. Subjects had to conduct two consecutive tasks on the target numbers, namely a comparison task and a parity decision task. We hypothesized that in the magnitude comparison task the first digit of the prime would primarily mediate priming effects because the tens digit has the largest predictive value when comparing magnitudes. In contrast, to make an accurate parity decision, one needs to attend to the unit position. Therefore, we predicted that priming effects in the parity task would mainly be caused by analysis of the second digit of the prime.

\section{Method}

\section{Participants}

Fourteen students (mean age $=19$ years; 2 males) of the University of Leuven participated as partial fulfillment of a course requirement. All participants were unaware of the purpose of the experiment.

\section{Stimuli and Procedure}

All targets were two-digit numbers ranging from 12 to 98 . Numbers with identical digits or comprising a zero were excluded, resulting in 72 target numbers. Seven priming conditions were created: one baseline condition (e.g., the prime-target pair \#\#_18), three prime conditions with a task-congruent overlap (e.g., 18_18, 1\#_18, and \#8_18), and three priming conditions with a task-incongruent overlap (e.g., 81_18, 8\#_18, and \#1_18).

Stimuli were presented on a 15 in. color screen with a vertical refresh cycle of $16.7 \mathrm{~ms}$. Each trial consisted of the following sequence of events. First, a forward mask was shown for $80 \mathrm{~ms}$. This mask consisted of four hash marks (\#\#\#). Then, the prime was presented for $50 \mathrm{~ms}$, followed by a backward mask (\#\#\#) for another $50 \mathrm{~ms}$. Finally, the target was presented for $200 \mathrm{~ms}$. All stimuli were presented centrally, corresponding to the positions of the two inner hash marks of the masks. After each trial, the screen was cleared and the next trial started after $1,200 \mathrm{~ms}$. In order to reduce physical overlap between prime and target, the primes (Arial font 11) were printed smaller than the targets and masks (Arial font 12). All stimuli were presented as white letters on a black background. Under these presentation conditions, at least some of the primes can be consciously detected. However, these presentation conditions suffice to ensure automatic priming effects. Moreover, previous number priming studies have shown that consciously perceiving the primes does not modulate the priming effects in a qualitative way, but only in a quantitative manner (Reynvoet \& Brysbaert, 2004).

Participants were asked to conduct two tasks: a magnitude comparison task (Experiment 1a) and a parity decision task (Experiment 1b). Decisions were made using the two outer buttons of a four-key response box. In the magnitude task, participants were instructed to indicate as quickly and as accurately as possible if the target number was smaller or larger than 55 by pressing the left button of the response box if the target was smaller than 55 and the right button if the target was larger than 55. In the parity task, participants were asked to indicate whether the target number was even or odd, by pressing either the left or right response button. The two tasks were performed consecutively in one session, with a short break between both tasks. The order of the tasks and the response assignment for both tasks were counterbalanced between participants.

Each task started with a practice block of 20 trials, randomly chosen from the entire set of all prime-target combinations. During the experimental session, all prime-target combinations (i.e., 72 Targets $\times 7$ Prime Conditions) were presented once for each task, resulting in a total of 504 trials. The entire session lasted about $1 \mathrm{hr}$.

\section{Results}

\section{Experiment 1a: Magnitude Task}

Inaccurate responses (on average 5.7\%) and responses slower than $1,200 \mathrm{~ms}$ or faster than $150 \mathrm{~ms}$ (on average $2.9 \%$ ) were discarded from all analyses. A repeated measures analysis was conducted with Prime Condition (seven levels: the seven prime types) as within-subject factor. Previous studies using magnitude comparison and parity judgment tasks demonstrated faster responses to trials where prime and target elicit the same response compared to trials where they elicit different responses (i.e., response congruency effect; e.g., Naccache \& Dehaene, 2001; Reynvoet, Caessens, \& Brysbaert, 2002). Therefore, we performed separate repeated measures analyses for compatible (i.e., both digits are either smaller or larger than 5 in the magnitude task or have the same parity status in the parity decision task, e.g., 13) and incompatible trials (i.e., one digit is smaller and the other is larger than 5 in the magnitude task or have the opposite parity status in the parity decision task, e.g., 18). The entire stimulus list can be found in the Appendix. Priming effects from positioncongruent primes should not be affected by this compatibility effect. In contrast, we expected stronger interference effects from position incongruent primes on incompatible targets than on compatible targets because in the case of incompatible targets (e.g., 81_18), prime and target elicit different responses whereas this is not the case with compatible targets (e.g., 31_13). ${ }^{1}$

\section{Compatible Targets}

The main effect of Prime Condition was significant, $F(6,78)=8.48, p<.001$. The mean RT for the baseline

1 The average absolute distance to the standard in the comparison task for compatible and incompatible targets was 22 and 22.5 , respectively. 
condition (\#\# 13) ${ }^{2}$ was $577 \mathrm{~ms}$. The mean RTs for the other six prime types were, respectively, $526 \mathrm{~ms}$ for 13 13, $542 \mathrm{~ms}$ for 1\#_13, $567 \mathrm{~ms}$ for \#3_13, $562 \mathrm{~ms}$ for 31_13, $553 \mathrm{~ms}$ for 3\#_13, and $586 \mathrm{~ms}$ for \#1_13 (see Figure 1). Planned comparisons between each prime type and the baseline (\#\# 13) revealed facilitation effects of 51 and $35 \mathrm{~ms}$ for the position-congruent prime conditions 13_13 and 1\#13 (respectively, $F(1,13)=29.27, p<.001$ and $F(1,13)=$ $32.60, p<.001$. In addition, a marginally significant facilitation effect of $24 \mathrm{~ms}$ was observed for the prime-incongruent condition 3\# 13, $F(1,13)=3.78, p<.08$. None of the other conditions differed significantly from baseline.

\section{Incompatible Targets}

The main effect of Prime Condition was significant, $F(6,78)=22.34, p<.001$. The mean RT for the baseline condition (\#\#_18) ${ }^{1}$ was $565 \mathrm{~ms}$. The mean RTs for the other six prime types were, respectively, $518 \mathrm{~ms}$ for $18 \_18$, $533 \mathrm{~ms}$ for $1 \# 18,567 \mathrm{~ms}$ for $\# 818,628 \mathrm{~ms}$ for $81^{-} 18$, $592 \mathrm{~ms}$ for $8 \# \_18$, and $575 \mathrm{~ms}$ for \#1_18 (see Figure 2). Planned comparisons between each prime type and the baseline (\#\# 18) showed facilitation effects of 47 and $32 \mathrm{~ms}$ for the position-congruent prime conditions $18 \_18$ and 1\# 18 (respectively, $F(1,13)=13.88, p<.01$ and $F(1,13)=9.00, p<.05)$. Contrary to the compatible trials, we also observed inhibitory priming effects of 63 and $27 \mathrm{~ms}$ for the position-incongruent priming conditions 81_18 and $8 \# 18$ (respectively, $F(1,13)=44.26, p<.001$ and $F(1,13)=13.23, p<.01)$. The other prime conditions were not significantly different from baseline.

\section{Experiment 1b: Parity Task}

Inaccurate responses (on average 6.9\%) and responses that fell outside the response window of $150-1,200 \mathrm{~ms}$ (on average $2.3 \%$ ) were discarded from all analyses. As before, separate repeated measures analyses with Prime Condition as within-subject factor were conducted for compatible and incompatible targets.

\section{Compatible Targets}

The main effect of Prime Condition was significant, $F(6,78)=9.51, p<.001$. The mean RT for the baseline condition (\#\#13) was 564 ms. The mean RTs for the other six prime types were, respectively, $514 \mathrm{~ms}$ for 13_13, $549 \mathrm{~ms}$ for $1 \#$ 13, $539 \mathrm{~ms}$ for \#3_13, $570 \mathrm{~ms}$ for $31_{-}^{-} 13$, $581 \mathrm{~ms}$ for $3 \#-13$, and $552 \mathrm{~ms}$ for \#1_13 (see Figure 1). Planned comparisons between each prime type and the baseline (\#\#_13) revealed significant facilitation effects of 50 and $25 \mathrm{~ms}$ for the position-congruent conditions 13 13 and \#3_13 (respectively, $F(1,13)=21.93, p<.001$ and $F(1,13)=6.14, p<.05)$. In addition, an inhibitory effect

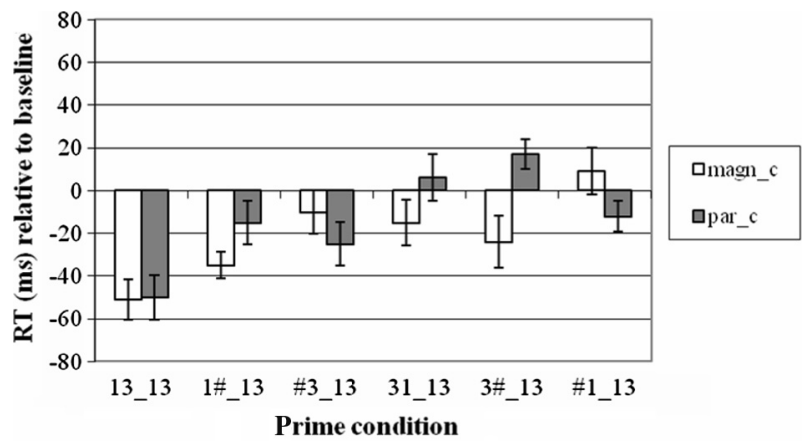

Figure 1. Average RTs on compatible targets for different priming conditions, relative to the baseline condition. Error bars represent one standard error above and below the mean. Magn_c = magnitude comparison task, compatible targets; par_c $=$ parity task, compatible targets.

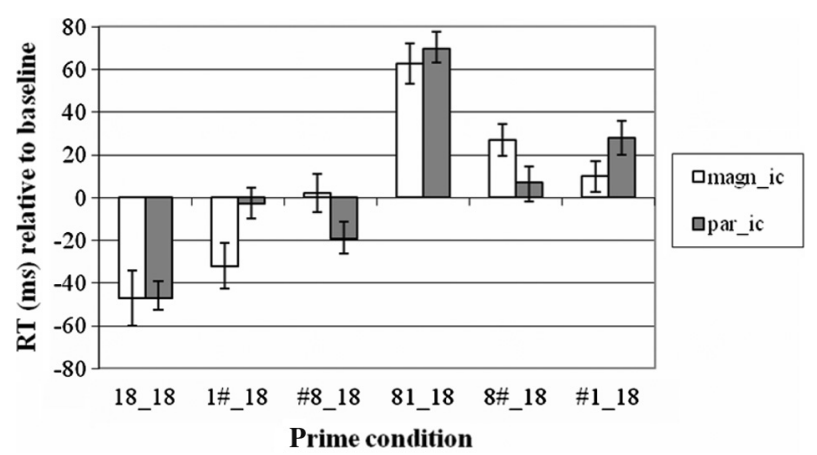

Figure 2. Average RTs on incompatible targets for different priming conditions, relative to the baseline condition. Error bars represent one standard error above and below the mean. Magn_ic = magnitude comparison task, incompatible targets; par_ic = parity task, incompatible targets.

of $17 \mathrm{~ms}$ for the position-incongruent condition 3\#13 was found, $F(1,13)=6.16, p<.05$. The other priming conditions were not significantly different from baseline.

\section{Incompatible Targets}

The main effect of Prime Condition was significant, $F(6,78)=37.56, p<.001$. The mean RT for the baseline condition (\#\#_18) was $575 \mathrm{~ms}$. The mean RTs for the other six prime types were, respectively, $528 \mathrm{~ms}$ for $18 \_18$, $572 \mathrm{~ms}$ for 1\#_18, $556 \mathrm{~ms}$ for \#8_18, $645 \mathrm{~ms}$ for 81_18, $582 \mathrm{~ms}$ for $8 \# 18$, and $603 \mathrm{~ms}$ for \#1_18 (see Figure 2). Planned comparisons between each prime type and the baseline (\#\#_18) showed a response facilitation of 47 and $19 \mathrm{~ms}$ for the prime-consistent conditions $18 \_18$ and \#8_18 (respectively, $F(1,13)=6.14, p<.05$ and

2 To illustrate the mean reaction times on the seven prime types, we use the example of the target " 13 " for compatible trials and target " 18 " for incompatible trials, but these reaction times were calculated across all stimuli within a prime type. 
$F(1,13)=79.82, p<.001)$. In addition, inhibitory effects of 70 and $28 \mathrm{~ms}$ for the position-inconsistent conditions 81_18 and \#1_18 were observed (respectively, $F(1,13)=$ $11 \overline{3} .15, p<.001$ and $F(1,13)=13.74, p<.01)$.

\section{Joint Analysis for Compatible and Incompatible Targets}

To examine the positional congruency effect in the parity judgment task in more detail, we conducted an additional analysis comparing four different types of priming. We divided the different priming conditions into (a) position-unspecific congruent priming in compatible trials, that is, compatible trials where the correct parity response is primed at the unit position (i.e., the previous compatible conditions 13_13, \#3_13, \#1_13, and 31_13), (b) positionspecific congruent priming in incompatible trials, that is, incompatible trials where the correct parity response is primed at the unit position (i.e., the previous incompatible conditions 18_18 and \#8_18), (c) position-specific neutral priming in incompatible trials, that is, incompatible trials where the prime does not trigger a parity response because only a decade digit is presented in the prime display (i.e., $8 \# 18$ and 1\# 18) and finally (d) position-specific incongruent priming, that is, incompatible trials where the unit digit triggers an incongruent parity response (i.e., 81_18 and $\# 1$ 18). Average RTs in these four conditions were 544, $54 \overline{2}, 577$, and $624 \mathrm{~ms}$, respectively. Paired $t$ tests indicated that there was no difference between position-specific congruent priming for compatible and incompatible targets, $t(13)=0.61, p=.55$. However, position-specific congruent priming led to faster RTs than position-specific neutral priming, $t(13)=4.66, p<.001$, which in turn was responded to faster than position-specific incongruent priming trials, $t(13)=6.33, p<.001$. These results show that position is a strong modulator of the priming effects: Facilitation is observed when the correct parity is primed at the relevant unit position and interference is observed when the incorrect parity is primed at the relevant unit position.

\section{Comparison Between Magnitude and Parity Tasks}

To investigate our a priori hypothesis, we compared the results of both tasks. Figures 1 and 2 already show that the priming effects in both tasks have a completely different pattern. Whereas facilitation/inhibition effects in the magnitude tasks are mediated by the prime number on the tens position, the priming effects in the parity task are mainly due to the prime number on the unit position. To explicitly test these differences between both tasks, we performed two new repeated measures analyses (separately for compatible and incompatible trials) with Task (two levels: magnitude and parity) as an additional within-subjects factor. For both analyses, we observed a significant interaction effect between Task and Prime Condition (for compatible trials: $F(6,78)=4.36, p<.001$; for incompatible trials:
$F(6,78)=4.01, p<.01$, indicating that task instructions mediated the priming effects.

\section{Discussion}

Previous research showed that two-digit numbers can be processed holistically and can be decomposed in a unit and decade number depending on presentation conditions, suggesting flexible processing mechanisms for such stimuli. In the present study, we further extended this idea by showing that the processing of two-digit numbers is also highly dependent on the informational value of each of its components. In a comparison task, priming effects are driven by the decade digit because this position is more informative in order to successfully complete this task. In contrast, in a parity decision task the priming effects are more influenced by the unit position, since the decision in this task needs to be based on that position. Furthermore, the finding that, either the decade or unit digit will be processed depending on the task instructions, suggests a flexible compositional processing style of the two-digit numbers.

In sum, this study clearly demonstrates that subjects rely on a very efficient processing mode for two-digit numbers and focus primarily on task-relevant components of the stimuli.

\section{Acknowledgments}

Bert Reynvoet is affiliated to the Laboratory of Experimental Psychology of the Department of Psychology, University of Leuven and to the Subfaculty of Psychology and Educational Sciences, University of Leuven, Campus Kortrijk. He is supported by OT/09/018 granted by the KU Leuven Research Council.

\section{References}

Cohen-Kadosh, R., \& Walsh, V. (2009). Numerical representation in the parietal lobes: Abstract or not abstract? Behavioural and Brain Sciences, 32, 313-328.

Dehaene, S., Dupoux, E., \& Mehler, J. (1990). Is numerical comparison digital? Analogical and symbolic effects in twodigit number comparison. Journal of Experimental Psychology: Human Perception and Performance, 16, 626-641.

Ganor-Stern, D., Pinhas, M., \& Tzelgov, J. (2009). Comparing two-digit numbers: The importance of being presented together. Quarterly Journal of Experimental Psychology, $62,444-452$.

Gazzellini, S., \& Laudanna, A. (2011). Digit repetition effect in two-digit number comparison. Zeitschrift für Psychologie / Journal of Psychology, 219, 30-36.

Moeller, K., Nuerk, H. C., \& Willmes, K. (2009). Internal number magnitude is not holistic either. European Journal of Cognitive Psychology, 21, 672-685.

Naccache, L., \& Dehaene, S. (2001). Unconscious semantic priming extends to novel unseen stimuli. Cognition, 80, 223-237. 
Nuerk, H.-C., Weger, U., \& Willmes, K. (2001). Decade breaks in the mental number line. Putting the tens and units back in different bins. Cognition, 82, B25-B33.

Reynvoet, B., \& Brysbaert, M. (1999). Single-digit and two-digit Arabic numerals address the same semantic number line. Cognition, 72, 191-201.

Reynvoet, B., \& Brysbaert, M. (2004). Cross-notation number priming investigated at different stimulus onset asynchronies in parity and naming tasks. Experimental Psychology, 51, 81-90.

Reynvoet, B., Caessens, B., \& Brysbaert, M. (2002). Automatic stimulus-response associations may be semantically mediated. Psychonomic Bulletin \& Review, 9, 107-112.

Reynvoet, B., \& Notebaert, K. (2009). Abstract or not? Insights from priming. Behavioral and Brain Sciences, 32, 349-350.

Van Opstal, F., Gevers, W., De Moor, W., \& Verguts, T. (2008). Dissecting the symbolic distance effect: Priming and comparison distance effects in numerical and non-numerical orders. Psychonomic Bulletin \& Review, 15, 419-425.

Verguts, T., \& De Moor, W. (2005). Two-digit comparison: Decomposed, holistic or hybrid? Experimental Psychology, 52, 195-200.
Zhang, W., \& Wang, H. (2005). The effect of external representations on numerical tasks. The Quarterly Journal of Experimental Psychology, 58, 817-838.

Zhou, X., Chen, C., Chen, L., \& Dong, Q. (2008). Holistic or compositional representation of two-digit numbers? Evidence from the distance, magnitude and SNARC effects in a number-matching task. Cognition, 106, 1525-1536.

Bert Reynvoet

Department of Psychology

University of Leuven

Campus Kortrijk

E. Sabbelaan 53

B-8500 Kortrijk

Belgium

Tel. +3256246177

E-mail bert.reynvoet@kuleuven-kortrijk.be

\section{Appendix}

\section{Stimuli Used in the Experiments}

Compatible targets in the comparison task

$12,13,14,15,21,23,24,25,31,32,34,35,41,42,43,45,51,52,53,54$,

$56,57,58,59,65,67,68,69,75,76,78,79,85,86,87,89,95,96,97,98$

Compatible targets in the comparison task

$16,17,18,19,26,27,28,29,36,37,38,39,46,47,48,49$,

$61,62,63,64,71,72,73,74,81,82,83,84,91,92,93,94$

Compatible targets in the parity decision task

$13,15,17,19,24,26,28,31,35,37,39,42,46,48,51,53$,

$57,59,62,64,68,71,73,75,79,82,84,86,91,93,95,97$

Incompatible targets in the parity decision task

$12,14,16,18,21,23,25,27,29,32,34,36,38,41,43,45,47,49,52,54$,

$56,58,61,63,65,67,69,72,74,76,78,81,83,85,87,89,92,94,96,98$ 